\title{
Gravidez na Adolescência: um Desafio à Promoção da Saúde Integral do Adolescente
}

\author{
Teenage Pregnancy: a Challenge for \\ Comprehensive Health Promotion Among \\ Adolescents
}

\author{
Marcella Furst Gonçalves Guanabens ${ }^{I}$ \\ Alessandra Miranda Gomes \\ Maria Elizete da Mata \\ Zilma Silveira Nogueira Reis ${ }^{I}$
}

\author{
PALAVRAS-CHAVE: \\ - Gravidez; \\ - Saúde do Adolescente; \\ - Aconselhamento Sexual; \\ - Educação; \\ - Assistência Integral à Saúde.
}

\section{KEYWORDS:}

- Pregnancy;

- Adolescent Health;

- Sex Counseling;

- Education;

- Comprehensive Health Care.

Recebido em: 01/10/2010

Aprovado em: 19/11/2010 $20 \frac{\text { REVISTA BRASILEIRA DE EDUCAÇĀO MÉDICA }}{36(1, \text { Supl. 2): 20-24; } 2012}$

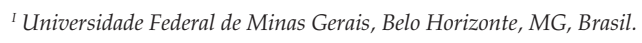

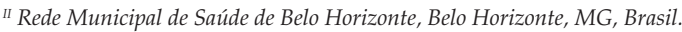




\section{INTRODUÇÃO}

A saúde sexual e reprodutiva dos adolescentes é motivo de constante preocupação para pais, educadores, profissionais de saúde e governantes, uma vez que suas consequências são de alto impacto individual e social. É fato que a associação entre conhecimento de métodos contraceptivos e prática do sexo seguro é frágil, levando à gravidez na adolescência e ao contágio de doenças sexualmente transmissíveis ${ }^{1}$. Mesmo em queda, a incidência de grávidas adolescentes no Brasil é considerada elevada, correspondendo a $21,6 \%$ do total de grávidas em $2006^{2}$. $\mathrm{O}$ acesso às políticas de prevenção e orientação sobre saúde sexual tem sido considerado de grande importância na redução do número de partos feitos em adolescentes na rede pública brasileira, que diminuiu em 30,6\% nos últimos dez anos ${ }^{3}$.

Um dos aspectos agravantes dessa questão é que a gravidez na adolescência é mais frequente nos estratos de renda mais baixa e, para muitas jovens, engravidar é uma escolha tomada como um meio de inserção social ${ }^{4}$. É notável, ainda, a relação existente entre gravidez e abandono escolar, pois se estima que $57,8 \%$ das meninas brasileiras com filhos não estudam nem trabalham ${ }^{4,5}$.

Para a promoção da saúde integral do adolescente, o Programa de Educação pelo Trabalho em Saúde (PET-Saúde) desenvolve ações integradas na comunidade do bairro São Marcos, região nordeste de Belo Horizonte, Minas Gerais. O grupo tutorial é formado por professor e alunos de graduação da Universidade Federal de Minas Gerais (UFMG) e trabalhadores da Secretaria Municipal de Saúde de Belo Horizonte. Para coordenar as ações de promoção da saúde é necessário que se faça, inicialmente, um diagnóstico local da saúde do adolescente. Esse passo inicial orienta o grupo tutorial e a Unidade Básica de Saúde (UBS) para atividades que realmente sejam capazes de causar impacto na melhoria das condições de saúde desse grupo etário. A área de abrangência dessa unidade de saúde possui população de aproximadamente 1.237 adolescentes ${ }^{6}$. Contudo, não se conhece ainda a dimensão do problema nessa comunidade ou mesmo entre as gestantes atendidas no pré-natal da UBS. O objetivo desse estudo é avaliar a dimensão do problema da gravidez na adolescência na área de atuação do grupo tutorial do PET-Saúde, por meio do levantamento e da análise de dados estatísticos secundários oficiais registrados no Sistema Datasus, do Ministério da Saúde.

\section{MÉTODOS}

Em um estudo observacional transversal, foi realizado um levantamento da prevalência de adolescentes grávidas na área de abrangência da UBS São Marcos. Foram utilizadas informações de domínio público cadastradas no Sistema de Acompanhamento do Programa de Humanização no Pré-Natal e Nascimento (SisPreNatal), integrante do Sistema Datasus, do
Ministério da Saúde. Empregou-se a definição cronológica de adolescência, compreendida entre os 10 e 19 anos de idade ${ }^{7}$. Nessa base de dados, os valores absolutos e relativos de adolescentes entre as grávidas registradas entre junho de 2009 a maio de 2010 foram buscados.

As complicações da gravidez na adolescência, abortamentos e partos prematuros registrados no Sinasc/Datasus foram levantadas no ano de 2009. A taxa de abortamento e de nascimentos prematuros entre as grávidas adolescentes e não adolescentes foi comparada, considerando-se apenas os nascimentos registrados na área de abrangência da UBS São Marcos, Belo Horizonte. A prematuridade foi definida como nascimento entre 22 e 37 semanas de gestação incompletas e abortamento, o produto da concepção expulso antes de 22 semanas. Para análise da associação entre gravidez na adolescência e as complicações gestacionais, prematuridade e abortamento, empregou-se o teste tipo quiquadrado (bicaudal) com utilização do teste exato de Fisher. A razão de chances e o intervalo de confiança de $95 \%$ foram obtidos para se estimar a chance de ocorrência da complicação no grupo de adolescentes no ano avaliado.

\section{RESULTADOS}

No período estudado, foram encontradas 149 gestantes registradas no SisPreNatal/Datasus que fizeram o acompanhamento da gravidez na UBS em questão. Entre elas, 33 (22,1\%) eram adolescentes. $\mathrm{O}$ percentual total de gestantes adolescentes registradas, assim como sua categorização por idade, é apresentado na tabela 1. Entre as adolescentes grávidas, 18,2\% eram pré-adolescentes (até 14 anos de idade), 36,4\% tinham entre 15 e 17 anos de idade, e $45,4 \%$ já eram maiores de idade (18 ou 19 anos - gráfico 1$)^{8}$.

\section{TABELA 1}

Distribuição etária das adolescentes grávidas da área de abrangência do grupo tutorial PET-Saúde

(UBS São Marcos - Belo Horizonte)

\begin{tabular}{ccc}
\hline $\begin{array}{c}\text { Idade } \\
\text { (anos) }\end{array}$ & $\begin{array}{c}\text { Número absoluto } \\
\text { de adolescentes } \\
\text { grávidas }\end{array}$ & $\begin{array}{c}\text { Grávidas adolescentes } \\
\text { em relação ao número total } \\
\text { de gestantes (\%) }\end{array}$ \\
\hline$<13$ & 0 & 0 \\
13 & 2 & 1,3 \\
14 & 4 & 2,7 \\
15 & 2 & 1,3 \\
16 & 3 & 2,0 \\
17 & 7 & 4,7 \\
18 & 10 & 6,7 \\
19 & 5 & 3,5 \\
\hline Total & 33 & 22,2 \\
\hline
\end{tabular}

Período avaliado: junho/2009 a maio/2010. Fonte: SisPreNatal (MS-BR). 


\section{GRÁFICO 1}

Distribuição, por idade, das 33 gestantes adolescentes da área de abrangência

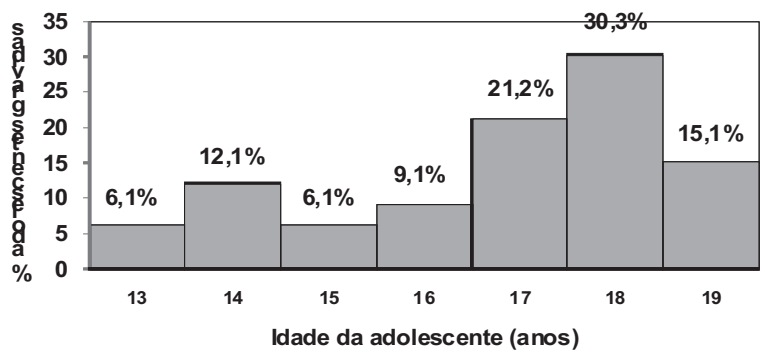

Nota: Período avaliado: junho/2009 a maio/2010. Fonte: SisPreNatal (MS-BR)

Os dados obtidos no Sinasc indicam que ocorreram 169 nascimentos na área de abrangência da UBS São Marcos no ano de 2009. Entre eles, 13 (7,7\%) nascimentos foram classificados como prematuros, sendo que quatro deles eram de mães adolescentes $^{2}$. Dessa forma, estima-se uma taxa de $12,1 \%$ de prematuros entre as adolescentes e de 6,6\% de nascimentos prematuros entre as grávidas não adolescentes (tabela 2). No entanto, não foi encontrada uma associação significativa entre a ocorrência de prematuridade registrada e adolescência nesse período $(\mathrm{p}=0,29)$. Foram encontrados, no mesmo sistema, 155 registros de egressos hospitalares no ano de 2009, sendo 148 $(95,5 \%)$ deles associados à gestação. O abortamento correspondeu a 10,1\% das causas de internação obstétrica da área de abrangência no ano de 2009 (tabela 2). Contudo, a ocorrência de abortamentos registrados entre adolescentes e não adolescentes não se mostrou estatisticamente diferente $(p=1,0)$.

\section{DISCUSSÃO}

A adolescência é reconhecida como uma etapa evolutiva da vida, compreendida entre a infância e a idade adulta. As características conflituosas naturais dessa fase envolvem transformações físicas, psicológicas e sociais que podem fragilizar os adolescentes de diferentes maneiras e intensidades, tornando- -os vulneráveis a uma série de riscos à saúde ${ }^{9}$. Aliadas à vulnerabilidade originada da impulsividade, pensamento mágico, imaturidade emocional e influência do grupo identificam-se questões sociais e econômicas como pontos fundamentais de desigualdade na questão da gravidez na adolescência, que é um problema nacional.

No Brasil, quase $18 \%$ das adolescentes do estrato de renda mais baixa são mães, enquanto no estrato de renda acima de cinco salários mínimos essa proporção não chega a 1\% ${ }^{4}$. A evasão escolar é também um fato, criando-se um círculo vicioso, pois a adolescente deixa os estudos para cuidar do filho, e o retorno à escola é dificultado, o que leva ao aumento dos riscos de desemprego, à dependência financeira dos familiares, à perpetuação da pobreza e da educação limitada ${ }^{4}$. Em Ribeirão Preto, São Paulo, um estudo caso-controle abrangendo 7.134 adolescentes grávidas apontou o predomínio de adolescentes envolvidas em atividades no lar e sem remuneração ${ }^{4}$. Embora essa questão ainda não seja objetivamente abordada no diagnóstico local de saúde deste grupo tutorial do PET-Saúde, a elevada prevalência de adolescentes entre as gestantes da área de abrangência chama atenção para a questão socioeconômica da comunidade como fator potencial de risco para esse problema. Isso realmente parece ser válido para Belo Horizonte, cidade na qual já foi constatado que áreas de maior vulnerabilidade socioeconômica, dentre elas a da UBS São Marcos, localizada na região nordeste, são aquelas em que se encontram as maiores taxas de nascidos vivos para mães com menos de 20 anos de idade. ${ }^{10}$

O perfil da adolescente grávida no período estudado mostra que quase a metade delas já eram maiores de idade (18 ou 19 anos), mas chama atenção para a constatação de uma situação grave, na qual 18,2\% eram pré-adolescentes (até 14 anos de idade). Dessa forma, espera-se que ações educativas do grupo tutorial possam ser planejadas para se iniciar precocemente, colocando em discussão questões como o projeto de vida, especialmente nas microrregiões de maior vulnerabilidade social, já mapeadas na área de abrangência. Nesses

TABELA 2

Complicações gestacionais entre adolescentes grávidas, na área de abrangência do grupo tutorial PET-Saúde (UBS São Marcos - Belo Horizonte)

\begin{tabular}{|c|c|c|c|c|c|}
\hline & $\begin{array}{l}\text { Grávidas } \\
\text { adolescentes } \\
\text { registradas }\end{array}$ & $\begin{array}{c}\text { Grávidas não } \\
\text { adolescentes } \\
\text { registradas }\end{array}$ & Total de grávidas & $\begin{array}{l}\text { Razão de chances } \\
\text { e IC } 95 \%\end{array}$ & $\mathrm{p}$ \\
\hline Nascimentos prematuros & $4 / 33(12,1 \%)$ & $9 / 136(6,6 \%)$ & $13 / 169(7,7 \%)$ & $1,95(0,47-7,61)$ & 0,29 \\
\hline Abortamentos entre egressos hospitalares & $3 / 31(9,7 \%)$ & $12 / 117(10,3 \%)$ & $15 / 148(10,1 \%)$ & $0,94(0,19-3,94)$ & 1,0 \\
\hline
\end{tabular}

Período avaliado: ano 2009. Fonte: Sinasc (MS-BR). 
locais, registram-se baixos níveis de escolaridade; domicílios precários; baixa renda; maiores taxas de homicídios; tráfico de drogas; entre outros tipos de violência.

Com relação às taxas de abortamento e de prematuridade entre os nascidos vivos de mães adolescentes avaliadas pelos dados do Sinasc/Datasus, observou-se que a adolescência não foi fator de agravamento para o mau resultado obstétrico, avaliado por esses dois indicadores, diferindo de outros estu$\operatorname{dos}^{11,12}$. Uma provável explicação para esse fato é que, mesmo não prevenindo a ocorrência da gravidez, a UBS tem sido acolhedora e eficiente em sua assistência pré-natal, proporcionado resultados obstétricos adequados. No entanto, não se pode esquecer a questão do abortamento inseguro, não registrado em dados oficiais, um dos responsáveis pela elevação da mortalidade materna nos países em desenvolvimento ${ }^{7}$.

Na adolescência, uma das principais transições é a passagem à sexualidade com parceiro. A insistência do homem e a resistência da mulher são comportamentos esperados. Os contatos corporais tornam-se mais íntimos e mais variados com o tempo, na medida em que é necessário manter o interesse do homem para prosseguir a relação ${ }^{13}$. Diferenças de atitudes masculinas e femininas entre os jovens são pontos reconhecidos, assim como a influência dos pais e dos profissionais de saúde na orientação para o sexo seguro ${ }^{14}$.

O presente estudo de reconhecimento da dimensão do problema nessa área de abrangência mostra que, entre junho de 2009 e maio de 2010, a prevalência de adolescentes entre as gestantes cadastradas no SisPreNatal $(22,2 \%)$ foi semelhante à prevalência média nacional oficial $(21,6 \% \text { em 2006) })^{2}$. Dessa forma, a realidade local não parece diferir da desconfortável situação brasileira. Um desafio a ser enfrentado pelo grupo tutorial, que pretende contribuir para reduzir esses índices nessa comunidade, deverá prever uma abordagem entre adolescentes de ambos os sexos, pais, educadores e profissionais, com ênfase na população mais carente. Dentre os prováveis motivos que podem levar ao desejo de engravidar estão: desejo inconsciente de ficar grávida; alternativa para sair de casa, da escola e ficar livre da pressão dos pais, contrariando ordens familiares; desejo de prender o namorado; carência afetiva; alívio da sensação de depressão e isolamento; desejo de ter mais poder, chamar a atenção para si; projeto de vida da adolescente, sendo uma escolha tomada como um meio de inserção social, visto que tal objetivo não é facilmente alcançado através de outros meios de condução à mobilidade social. ${ }^{15}$

Atentos para a importância do reconhecimento das questões mais relevantes para a saúde dos adolescentes, o grupo tutorial sinaliza a necessidade de aprofundamento da análise para permitir ações preventivas e educativas oportunas.
O PET-Saúde tem sido uma experiência de interação ensino-serviço-pesquisa de grande valor para a formação de alunos de graduação da UFMG. O reconhecimento dos problemas de saúde e sua interface com as questões socioeconômicas contribui para o entendimento do SUS e de sua dinâmica como espaço de atuação criativa e de produção de conhecimento. Para os profissionais de saúde, tem sido uma oportunidade de formação em serviço, colocando-os em contato com a complexidade da produção científica associada à consequente melhora na assistência. Para a comunidade, um SUS mais atuante e efetivo proporcionará uma resposta mais adequada às suas reais necessidades.

Apoio: Programa de Educação pelo Trabalho em Saúde (PET-Saúde). Departamento de Gestão da Educação na Saúde - Deges, da Secretaria de Gestão do Trabalho e da Educação na Saúde - SGTES, do Ministério da Saúde, com suporte do Sistema de Informações Gerenciais - SIG PET-Saúde, desenvolvido por técnicos do Departamento, em parceria com o Datasus/ SE/MS e o Fundo Nacional de Saúde/SE/MS.

\section{REFERÊNCIAS}

1. Yazlle MEHD. Gravidez na Adolescência. Rev. Bras. Ginecol. Obstet. [on-line]. Ago 2006. 28 (8) [Capturado em: 19 set. 2010]. Disponível em: http: //www.scielo.br/scielo. php?pid=S0100-72032006000800001\&script=sci_arttext.

2. Brasil. Ministério da Saúde do Brasil. Indicadores e Dados Básicos - Brasil. 2008. IDB-2008. Ministério da Saúde/SVS - Sistema de Informações sobre Nascidos Vivos (Sinasc).

3. Brasil. Ministério da Saúde do Brasil. Brasil acelera redução de gravidez na adolescência. [Capturado em: 19 set. 2010]. Disponível em: http: //portal.saude.gov.br/portal/aplicacoes/noticias/default.cfm?pg=dspDetalheNoticia\&id_ area $=124 \& C O \_$NOTICIA $=11137$.

4. Brasil. Instituto de Pesquisa Econômica Aplicada - Ipea. Fontoura NO, Pinheiro LS. Gravidez na adolescência. [Capturado em: 19 set. 2010]. Disponível em: http: // desafios2.ipea.gov.br/sites/000/17/edicoes/60/pdfs / rd60art04.pdf.

5. Michelazzo D, Yazlle MEHD, Mendes MC, Patta MC, Rocha JSY, Moura MD. Indicadores sociais de grávidas adolescentes: estudo caso-controle. Rev. Bras. Ginecol. Obstet. [on-line]. 2004. 26 (8) [Capturado em: 19 set. 2010]; 633-639. Disponível em: http: //www.scielo.br/pdf/rbgo/v26n8/ a07v26n8.pdf

6. Contagem feita a partir dos registros populacionais realizados pelos Agentes Comunitários de Saúde do Centro de Saúde São Marcos. 
7. World Health Organization. Adolescent pregnancy, issues in adolescent health and development. [Capturado em: 19 set. 2010]. Disponível em: http: / /whqlibdoc.who.int/publications/2004/9241591455_eng.pdf.

8. Brasil. Ministério da Saúde do Brasil. Sistema de Acompanhamento do Programa de Humanização no Pré-Natal e Nascimento (SisPreNatal).

9. Secretaria Municipal de Saúde de Belo Horizonte - PBH. Plano Municipal de Saúde de Belo Horizonte (2005-2008). [Capturado em: 19 set. 2010]. Disponível em: http: // www.enf.ufmg.br/internatorural/textos/Manuais/planomunicipaldesaude20052008.pdf.

10. Nogueira MJ, Silva BFA, Barcelos SM, Schall VT. Análise da distribuição espacial da gravidez adolescente no município de Belo Horizonte/MG. Rev. Bras. Epidemiol. [on-line]. 2009. 12(3). [Capturado em: 19 set. 2010]; 297312. Disponível em: http: //www.scielo.br/pdf/rbepid/ v12n3/02.pdf.

11. Tauil PL, Chaloult E, Rodrigues FRA, Nogueira LSC, Costa PETF, Santos SF. Gravidez em adolescentes: aspectos relativos ao pré-natal, parto e recém-nascido. Brasília Med. [on-line]. 2001. 38 (1/4). [Capturado em: 19 set. 2010]; 7-12. Disponível em: http: / /bases.bireme.br/cgi-bin/wxislind. exe/iah/online/?IsisScript=iah/iah.xis\&src=google\&bas $\mathrm{e}=$ LILACS\&lang=p\&nextAction=lnk\&exprSearch $=349172$ \&indexSearch=ID.

12. Suzuki CM, Ceccon MEJ, Falcão MC, Vaz FAC. Análise comparativa da frequência de prematuridade e baixo peso entre filhos de mães adolescentes e adultas. Rev. Bras. Crescimento Desenvolv. Hum. [on-line]. set-dez 2007. 17(3). [Capturado em: 19 set. 2010]; 95-10. Disponível em: http: / / www.revista-
susp.sibi.usp.br/scielo.php?script=sci_arttext\&pid=S0104$-12822007000300010 \& \operatorname{lng}=$ pt\&nrm=iso\&tlng=pt.

13. Brasil. Ministério da Saúde. Fundação Oswaldo Cruz. Um olhar sobre o jovem no Brasil - Brasília: Editora do Ministério da Saúde, 2008.

14. Matos EB, Veiga RT, Reis ZSN. Intenção de uso de preservativo masculino entre jovens estudantes de Belo Horizonte: um alerta aos ginecologistas. Rev. Bras. Ginecol. Obstet. [on-line]. 2009. 31(11). [Capturado em: 19 set. 2010]; 574-80. Disponível em: http: //www.scielo.br/pdf/rbgo/ v31n11/v31n11a08.pdf.

15. Cavasin S, Arruda S. Gravidez na adolescência: desejo ou subversão? [Capturado em: 19 set. 2010]. Disponível em: bvsms.saude.gov.br/bvs/publicacoes/156_04PGM2.pdf.

\section{CONTRIBUIÇÃO DOS AUTORES}

Marcella Guanabens e Alessandra Gomes participaram na revisão bibliográfica, coleta de dados, análise estatística e redação. Maria Elizete da Mata coordenou a coleta de dados e da redação. Zilma Reis participou do estudo, análise estatística e revisão

\section{CONFLITO DE INTERESSES}

Declarou não haver.

\section{ENDEREÇO PARA CORRESPONDENCIA}

Marcella Furst Gonçalves Guanabens

Rua José Barsand de Leucas, 205/201

Palmares - Belo Horizonte

CEP. 31160-530 MG

E-mail: marcellafurst@gmail.com 